\title{
Neural predictors of sensorimotor adaptation rate and savings
}

Kaitlin Cassady ${ }^{1}$, Marit Ruitenberg ${ }^{2 \S}$, Vincent Koppelmans ${ }^{2}$, Patricia Reuter-Lorenz ${ }^{1}$, Yiri De Dios ${ }^{3}$, Nichole Gadd ${ }^{3}$, Scott $W o o d^{4}$, Roy Riascos Castenada ${ }^{5}$, Igor Kofman ${ }^{3}$, Jacob Bloomberg $^{4}$, Ajitkumar Mulavara ${ }^{3}$, and Rachael Seidler ${ }^{1,2,6^{*}}$

${ }^{1}$ Department of Psychology, University of Michigan, Ann Arbor, MI, USA;

${ }^{2}$ School of Kinesiology, University of Michigan, Ann Arbor, MI, USA;

${ }^{3}$ KBRwyle Science, Technology, and Engineering Group, Houston, TX, USA;

${ }^{4}$ NASA Johnson Space Center, Houston, TX, USA;

${ }^{5}$ University of Texas Health Science Center, Houston, TX, USA;

${ }^{6}$ Neuroscience Graduate Program, University of Michigan, Ann Arbor, MI, USA;

$\S$ Current affiliation:

Department of Experimental Psychology, Ghent University, Ghent, Belgium

* Corresponding author

\section{Rachael Seidler}

University of Michigan, School of Kinesiology, Neuromotor Behavior Lab

401 Washtenaw Avenue, Ann Arbor, MI 48109-2214, USA

Email: rseidler@umich.edu

This is the author manuscript accepted for publication and has undergone full peer review but has not been through the copyediting, typesetting, pagination and proofreading process, which may lead to differences between this version and the Version of record. Please cite this article as doi:10.1002/ hbm.23924. 
In the present study, we investigate whether individual variability in the rate of visuomotor adaptation and multi-day savings is associated with differences in regional gray matter volume and resting state functional connectivity. Thirty-four participants performed a manual adaptation task during two separate test sessions, on average 9 days apart. Functional connectivity strength between sensorimotor, dorsal cingulate, and temporoparietal regions of the brain was found to predict the rate of learning during the early phase of the adaptation task. In contrast, default mode network connectivity strength was found to predict both the rate of learning during the late adaptation phase as well as savings. As for structural predictors, greater gray matter volume in temporoparietal and occipital regions predicted faster early learning, whereas greater gray matter volume in superior posterior regions of the cerebellum predicted faster late

learning. These findings suggest that the offline neural predictors of early adaptation may facilitate the cognitive aspects of sensorimotor adaptation, supported by the involvement of temporoparietal and cingulate networks. The offline neural predictors of late adaptation and savings, including the default mode network and the cerebellum, likely support the storage and modification of newly acquired sensorimotor representations.

Keywords: functional connectivity, gray matter volume, neural predictors, savings, sensorimotor adaptation 


\section{Introduction}

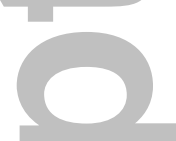

Sensorimotor adaptation is a gradual process of adjusting motor representations to remain engaged in goal-directed behavior following changes in the environment, sensory inputs, or body physical characteristics. Such adaptation has been studied by having participants adapt movements to, for example, visual perturbations (Bock 1992; Clower et al. 1996; Pine et al. 1996; Bock and Burghoff 1997; Inoue et al. 1997; Ghilardi et al. 2000; Imamizu et al. 2000; Krakauer et al. 2000) or force field perturbations (Shadmehr and Mussa-Ivaldi 1994; Shadmehr and Holcomb 1997). The early phase of sensorimotor adaptation is thought to rely mainly on cognitive processes, such as working memory, error detection and correction, and attention (Anguera et al. 2010; Taylor et al. 2014). In contrast, the later phase of adaptation is assumed to primarily involve slower and more implicit procedural processes (Seidler et al. 2006; Smith et al. 2006).

Previous studies have shown that individual differences in the rate of sensorimotor adaptation are associated with variability in task-based brain activation patterns. Specifically, faster learning during the early adaptation phase has been associated with greater activation in the right dorsolateral prefrontal cortex (Anguera et

al. 2011), as well as greater activation in cingulate, visual, and parietal cortices (Seidler et al. 2006). In addition to such functional, online predictors of adaptability, offline brain structural properties have also been linked to individual variation in sensorimotor adaptation. Della-Maggiore et al. (2009) found that the rate of visuomotor adaptation was positively correlated with fractional anisotropy in white-matter tracts connecting the cerebellum with motor and premotor cortices. These studies suggest that individual 
differences in both functional and structural neural characteristics could serve as predictors of adaptability, and help to identify brain networks involved in adaptation.

The adjustment of motor representations following adaptation can lead to motor memories that outlast the training session, as evidenced by observations that participants adapt faster when they have been previously exposed to the same perturbation. Such savings of adaptation have been observed immediately following initial learning (Seidler and Noll 2008; Bédard and Sanes 2011; Villalta et al. 2015), one month later (DellaMagiore and McIntosh 2005), and even one year after initial learning (Landi et al. 2011). To date, only few studies have investigated the neural mechanisms underlying multi-day savings of adaptation. Landi et al. (2011) explored how structural brain changes contributed to long-term memory of a visuomotor adaptation task. They found that a single week of adaptation training led to an increase in gray matter volume in contralateral primary motor cortex. Interestingly, the extent of this increase was found to predict long-term savings: participants who exhibited a larger increase in gray matter volume after one week of training showed more savings one year later. Della-Maggiore et al. (2015) found that resting-state functional connectivity within a sensorimotor network, including motor, premotor, and posterior parietal cortices, in addition to cerebellum and putamen, increased after a visuomotor adaptation task. Further, the magnitude of the connectivity increase within this network was found to predict savings 24 hours after initial learning. These studies provide evidence that structural and functional changes induced by visuomotor adaptation may have a long-term impact on behavior.

In the present study, we evaluated whether offline functional connectivity and structural neural characteristics can serve as predictors of adaptability and multi-day 
savings. In particular, we investigated whether individual differences in the rate of visuomotor adaptation and savings of adaptation over time are associated with differences in resting state functional connectivity (rs-fcMRI) and regional gray matter volume (using voxel-based morphometry; VBM). Participants performed a manual adaptation task in which they used a joystick to hit targets presented on a screen. After first performing the task under normal visual feedback, they then adapted to $45^{\circ}$ clockwise-rotated feedback. Participants performed the task during two separate test sessions, on average 9 days apart.

We hypothesized that faster learning during the early phase of adaptation would be predicted by stronger functional connectivity strength between sensorimotor areas within other frontal and parietal regions of the brain (including dorsolarteral prefrontal cortex (DLPFC), anterior cingulate cortex (ACC) insula and precuneus cortex) as taskbased activation in these regions has previously been shown to predict adaptability (Di Martino et al. 2008; Anguera et al. 2010; Anguera et al. 2011). Given the contribution of the superior posterior fissure region of the cerebellum during the late adaptation phase (Flament 1996; Imamizu et al. 2000; Seidler and Noll 2008), we further expected that faster learners during this stage would show stronger baseline connectivity between this cerebellar region with motor cortical areas. Based on previously reported associations between structural brain properties and sensorimotor adaptability (Della-Maggiore 2009), we expected that faster learning during the early adaptation phase would be associated with larger gray matter volume in sensorimotor cortical areas and frontoparietal regions (including DLPFC, ACC, insula and precuneus cortex). In contrast, we predicted that 
faster learning during the late adaptation phase would be associated with larger gray matter volume in superior posterior regions of the cerebellum and motor cortical areas.

With respect to multi-day savings we were interested in identifying networks in

which connectivity strength was correlated with individual differences in the extent of savings, as well as areas in which gray matter volume was correlated with such differences. Based on the findings from previous studies investigating the neural mechanisms underlying savings (Seidler and Noll 2008; Landi et al. 2011; DellaMaggiorre et al., 2015), we hypothesized that more savings would be predicted by stronger functional connectivity strength between superior posterior regions of the cerebellum with motor cortical areas and larger gray matter volume in these same regions (i.e., overlapping neural predictors between late learning and savings). However, as behavioral evidence has shown that savings is associated with early learning processes (Morehead et al. 2015; Haith et al. 2015), we may alternatively observe overlapping neural predictors between early learning and savings.

\section{Materials and Methods}

\section{Participants}

The present study included 34 healthy participants (30 male, 4 female; age $35.4 \pm$ 8.1 years) who each performed a visuomotor adaptation task at two separate test sessions, completed on average $9.3 \pm 6.5$ days apart. As 14 participants completed a different experimental protocol during the second session, their data were not included in the 
analyses that involved savings of adaptation from session 1 to session 2 . The analyses for test session 2 were thus based on data from 20 participants (16 male, 4 female, age $36.3 \pm$ 9.2). All participants were recruited via the Test Subject Facility at NASA Johnson Space Center and passed a modified Air Force class III physical. The Test Subject Facility at NASA Johnson Space Center (JSC) provides qualified test subjects for ground-based research or microgravity studies. The Test Subject Screening personnel at JSC recruit subjects that include employees from JSC and from outside of JSC. The facility does not select a particular type of subject population; subjects are only required to pass the physical. The modified class III physical is a medical examination that is required to enter the Air Force. All participants at the Test Subject Facility at NASA Johnson Space Center must pass this examination in order to participate in the study. All except four participants were right-handed. They reported average gaming experiences of 1.09 with 0 indicating "none at all" and 4 indicating "extreme" experience. A detailed explanation of the current study was provided to the participants, and all gave written informed consent. The study was approved by the Institutional Review Boards of the University of Michigan, the University of Texas - Medical Brach (UTMB), and NASA Johnson Space Center.

\section{Experimental Design and Procedure}

The manual sensorimotor adaptation task employed in the current study has been used extensively in previous work from our lab and others (e.g., Krakauer et al. 2000; Seidler et al. 2006; Anguera et al. 2007). For this task, participants lay supine in the MRI 
scanner and controlled a custom-built MRI-compatible dual axis joystick with the thumb and index finger of their right hand to hit targets presented on a screen (viewed via a mirror). Real-time feedback of the joystick location was presented as a cursor on the screen, using a scaling factor of 1 . Each movement was initiated from the central position on the screen. A target was presented every $2.5 \mathrm{~s}$, either $4.8 \mathrm{~cm}$ to the right, left, above, or below the central position.

Participants were instructed to move the cursor to the target as quickly as possible by moving the joystick, and to hold the cursor within the target until it disappeared. They were then instructed to release the joystick handle after target disappearance, allowing the spring-loaded joystick to re-center for the next trial. Participants performed four runs of experimental trials. The first run included two baseline blocks (B1 and B2) of 16 trials each under normal visual feedback. The next two runs included four blocks of 16 trials each under $45^{\circ}$ clockwise rotated feedback (blocks A1 - A8). The final run (blocks B3 and B4) was identical to the first run, which allowed us to calculate the aftereffects of adaptation, or 'readaptation'. Each 16-trial block alternated with 20s of visual fixation. This block design was repeated at two different test dates, allowing us to evaluate early and late adaptation, the aftereffects of adaptation, and savings of adaptation across days (see Figure 1). The second session was completed on average $9.3 \pm 6.5$ days after the initial session.

-

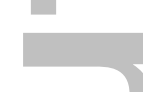

(Enter Figure 1 here)

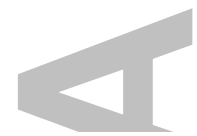

Resting state fMRI data acquisition

John Wiley \& Sons, Inc. 
For 18 of the participants, multi-sequence MRI was acquired using a $3.0 \mathrm{~T}$

Siemens Magnetom skyra MRI scanner located at UTMB at Galveston. We used a 3D T1 sagittal MP-RAGE sequence with the following parameters: $(\mathrm{TR}=1900 \mathrm{MS}, \mathrm{TE}=2.49$

MS, flip angle $=9^{\circ}, \mathrm{FOV}=270 \times 270 \mathrm{~mm}$, slice thickness $=0.9 \mathrm{~mm}, 192$ slices;

matrix $=288 \times 288$, voxel size $=0.94 \times 0.94 \mathrm{~mm}$, duration $=\sim 4$ minutes $)$. For rs-fcMRI, we used a single-shot gradient-echo (GRE) echo planar imaging (EPI) sequence to acquire $164 \mathrm{~T} 2 *$-weighted BOLD images $\left(\mathrm{TR}=3660 \mathrm{MS}, \mathrm{TE}=39 \mathrm{MS}\right.$, flip angle $=90^{\circ}$, FOV $=240 \times 240 \mathrm{~mm}$, slice thickness of $4 \mathrm{~mm}, 1 \mathrm{~mm}$ slice gap, matrix $=94 \times 94$, voxel size $=2.55 \times 2.55 \times 5.0 \mathrm{~mm}, 36$ axial slices, duration $=\sim 10$ minutes $)$.

For 16 of the participants, fMRI data were collected on a $3.0 \mathrm{~T}$ Siemens Magnetom Verio, located at UTMB Victory Lakes. For these participants, we used a 3D T1 sagittal MP-RAGE sequence with the following parameters: $(\mathrm{TR}=1900 \mathrm{~ms}, \mathrm{TE}=2.32$ ms, flip angle $=9^{\circ}, \mathrm{FOV}=250 \times 250 \mathrm{~mm}$, slice thickness $=0.9 \mathrm{~mm}, 192$ slices; matrix $=512 \times 512$, voxel size $=0.49 \times 0.49 \mathrm{~mm}$, duration $=\sim 4$ minute $)$. For T1 preprocessing, the in-plane resolution was down sampled to $0.94 \mathrm{x} 0.94 \mathrm{~mm}$. For rs-fcMRI, we used a single-shot gradient-echo (GRE) echo planar imaging (EPI) sequence to acquire $164 \mathrm{~T} 2 *$-weighted BOLD images $\left(\mathrm{TR}=3660 \mathrm{MS}, \mathrm{TE}=39 \mathrm{MS}\right.$, flip angle $=90^{\circ}$, $\mathrm{FOV}=250 \times 250 \mathrm{~mm}$, slice thickness of $4 \mathrm{~mm}, 1 \mathrm{~mm}$ slice gap, matrix $=94 \times 94$, voxel size $=2.66 \times 2.66 \times 5.0 \mathrm{~mm}, 36$ axial slices, duration $=\sim 10$ minutes $).$ All participants were instructed to keep their eyes open, to remain awake, and to look at a fixation point while not thinking about anything in particular during the resting state fMRI acquisition. Due to differential MRI scanners, scanner sequences and staff, scanner site was used as a 
covariate in all analyses.

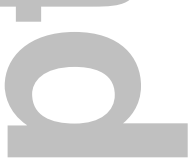

Behavioral data processing and analyses

Direction error (DE) was measured in order to examine performance during adaptation. This was defined as the angle between the line connecting the start and target positions (in joystick coordinates) and the line connecting the start with the spatial location of the joystick at the time of peak velocity (see Figure 2). Trials for which DE deviated more than 2.5 standard deviations from the mean across a test session were replaced by the mean of the directly preceding and succeeding trials. This was computed separately for each of the two sessions per participants and resulted in the replacement of $2.38 \%$ of the trials overall. Movement time (MT) and reaction time (RT) were also measured for each trial. MT was defined as the time it took for participants to move the cursor from the start to the target position, whereas RT was defined as the time it took for participants to begin moving the cursor once a new trial began.

\section{(Enter Figure 2 here)}

\section{(}

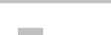

A repeated measures analysis of variance (ANOVA) was performed on each outcome measure with session (2), block (12), and trial (16) defined as the within-subject variables. As aforementioned this analysis was performed on the data from the 20 participants that completed identical protocols on both test sessions. For all ANOVAs, 
the Huynh-Feldt correction (Huynh and Feldt, 1970) was applied when the assumption of sphericity was not met. The threshold for statistical significance was set at $p<0.05$.

Each participant's rate of learning during the first test session was determined by computing the exponential decay constant across adaptation trials. We used MATLAB's fit function to fit a single-term exponential model to early learning and late learning data for all participants, with the initial value specified as "[0,0]". All other parameters were default to the function. This decay constant was used as the primary outcome measure for examining functional connectivity predictors of sensorimotor adaptation, with more negative values reflecting faster adaptation. Several previous studies have demonstrated that visuomotor adaptation data are generally well-characterized by exponential decay functions (Flanagan et al., 1999; Krakauer et al., 2000; Krakauer et al., 2005; Burge, 2008; King et al., 2009). To confirm that such exponential functions better characterized the present data for early and late learning than linear functions, we measured the $\mathrm{R}^{2}$ fits using both approaches. Indeed, we found that exponential functions provide significantly better fits than linear functions, for both early and late learning (early: $t=6.20, p<.001$; late: $t=5.99, p<.001)$. Example single subject DE data and exponential decay fits are illustrated in Figure S1 (in the supplementary materials).

We differentiated the rate of learning during the early, late, and post adaptation phases by assigning adaptation blocks A1-A4 as "early", adaptation blocks A5-A8 as "late", and "post adaptation" baseline blocks as B3 and B4. Finally, to assess multi-day savings, we computed individual savings scores. Specifically, savings was measured as the difference between a participant's mean DE in the first adaptation block (i.e., the first 16 trials with rotated feedback) on the first test session and the mean DE in that block on 
the second test session. It should be noted that learning rates and savings scores thus were calculated on an individual basis.

Subsets of these data have already been published in part by Ruitenberg et al.

(2017) in a comparison of manual and locomotor adaptation. Here, we present the manual adaptation results for a larger group of participants, and for the first time focus on associations between behavior and rs-fcMRI as well as VBM measures.

\section{Resting state fMRI data preprocessing}

Rs-fcMRI data were first corrected for slice timing using sinc interpolation and then realigned for head motion correction using statistical parametric mapping software (SPM8, Welcome Department of Imaging Neuroscience, Institute of Neurology, London, United Kingdom). To examine outliers due to spiking and movement, we used the Artifact Detection Tool (ART) software package (Whitfield-Gabrieli 2009). Next, whole brain rs-fcMRI images were normalized to MNI152 space using a multi-step procedure. First, the T1 image was corrected for field inhomogeneities using N4ITK within an intracranial mask that was obtained using FSL's brain extraction tool (BET; Tustison et

al. 2010). The bias field corrected and averaged image was skull stripped using FSL's

BET using robust brain center estimation and a fractional intensity threshold of 0.1 .

SPM8 was used to coregister the skull stripped bias field corrected T1 image to the mean rs-fMRI EPI. The co-registered images were normalized to MNI152 common space using advanced normalization tools (ANTs) with cross correlation as the similarity metric and symmetric normalization as the transformation model (Avants et al. 2011). 
The resulting warp parameters were applied to the 4D EPI images that were subsequently smoothed with a Gaussian kernel of $4 \mathrm{~mm}$ sigma ( $9.4 \mathrm{~mm}$ FWHM). To maximize cerebellar normalization accuracy, we isolated the cerebellum using the SUIT toolbox and registered the isolated cerebellum to the MNI152 cerebellum that was normalized to SUIT space (Diedrichsen 2006). The normalization steps for the cerebellum were then identical to the ones for the whole brain described above.

\section{Functional connectivity analyses}

Functional connectivity MRI (fcMRI) analyses were performed using the CONN toolbox (Whitfield-Gabrieli and Nieto-Castanon 2012). The rs-fcMRI data were filtered using a temporal band-pass filter of .008 to $.09 \mathrm{~Hz}$ in order to examine the frequency band of interest and to exclude higher frequency sources of noises such as heart rate and respiration. Linear detrending was used to remove any linear trends within each

functional run. For noise reduction, we used an anatomical component-based noise correction method called aCompCor, which models the influence of noise as a voxelspecific linear trend combination of multiple empirically estimated noise sources by extracting principal components from noise regions of interest (ROIs) and including them as nuisance parameters in the general linear models (Behzadi et al. 2007). Specifically, the anatomical images for each participant were segmented into white matter (WM), gray matter (GM), and cerebrospinal fluid (CSF) masks using the default parameters of the SPM8 Segment function (Ashburner and Friston, 2005). These parameters include a nonlinear deformation estimated field that best overlays the tissue probability maps on each participants' image. The model is further refined by allowing tissue probability maps to be deformed based on a set of estimated parameters. This 
allows spatial normalization and segmentation to be combined into the same model. This procedure uses a low-dimensional approach, which parameterizes the deformations by a linear combination of about one thousand cosine transform bases.

To reduce partial voluming with GM, the WM and CSF masks were eroded by one voxel. The eroded WM and CSF masks were then used as noise ROIs. Time courses from all ROIs were extracted from the unsmoothed functional volumes to avoid risk of potential "spillage" of the BOLD signal from nearby regions. Residual head motion parameters (three rotations and three translations, in addition to six parameters that represent their first-order temporal derivatives) and signals from WM and CSF were regressed out during the computation of functional connectivity maps.

For statistical analyses, we performed both hypothesis driven (seed-to-voxel) and hypothesis-free (voxel-to-voxel) approaches. For the first-level seed-to-voxel analysis, we selected ten ROIs to examine for brain-behavior associations. These ROIs were based on results from previous studies investigating associations between brain activation and early/late adaptation and savings (see Table 1). Cortical ROIs were defined as $6 \mathrm{~mm}$ radius spheres and subcortical ROIs as $4 \mathrm{~mm}$-radius spheres, centered on peak coordinates taken from the literature. The mean time series of each ROI was obtained by averaging the time series of all voxels within that region. Next, Pearson's correlation coefficients were computed between the mean time series of each ROI and the time series of each voxel in an a standard a priori brain mask. This mask refers to a standard MNI-space brain mask used for voxel-based analyses only, and limits analyses only to voxels within the mask. 
First-level voxel-to-voxel analysis included the calculation of voxel-to-voxel functional correlation matrices for each participant. From the residual BOLD time series at each voxel with an a priori brain mask, the matrix of voxel-to-voxel bivariate correlation coefficients was computed. From this correlation matrix, the intrinsic connectivity contrast defining the overall strength of the global connectivity patterns (Martuzzi et al. 2011) was computed between each voxel and all other voxels in the brain. This approach was employed to assess network changes that may not have been identified with our hypothesized ROIs. For both seed-to-voxel and voxel-to-voxel analyses, the correlation coefficients were converted into z-values using Fisher's r-to-z transformation in order to improve their normality.

\section{(Enter Table 1 here)}

$(\mathrm{nan}=0$

Positive and negative associations between voxel-to-voxel fcMRI and behavioral performance were examined using one-sample t-tests in SPM8. In this model, first level beta maps were included as the main variables of interest. Each participant's rate of early and late learning as well as aftereffects and savings were included as covariates. Statistical significance was determined with a cluster-level FDR $p<0.05$ to correct for multiple comparisons. For the seed-to-voxel second level analysis, one subject-averaged mask was created for the regions correlated with each ROI during session 1 (using a cluster-level FDR $\mathrm{p}<0.05$ to correct for multiple comparisons). From these masks, one average functional connectivity measure was extracted for each network and each participant. To examine the relationship between these fcMRI measures and performance, 
Pearson's correlation coefficient was computed across each participant's average fcMRI measure and their rates of early and late learning, aftereffects and savings.

\section{Voxel-based morphometry}

Whole-brain VBM analyses were performed using the VBM8 toolbox for SPM8 (http://dbm.neuro.uni-jena.de/vbm.html). Cortical thickness analysis can provide additional information to a VBM analysis, and these different measures can be sensitive to different pathologies. However, these measures are correlated (Hutton et al., 2009), which is why we chose to report on only one of these two measures. VBM reflects cortical thickness, cortical surface area, and gyral folding, whereas cortical thickness only reflects the thickness (Hutton et al., 2009). Furthermore, cortical thickness analysis excludes the deep gray matter areas, which are important in sensorimotor learning. For these reasons, we selected VBM over cortical thickness analysis.

High-resolution T1-weighted anatomical images were segmented, modulated using the non-linear DARTEL warping parameters from the normalization results, and then smoothed with a standard deviation of $8 \mathrm{~mm}$ full width at half maximum. Positive and negative associations between gray matter volume and sensorimotor adaptation metrics were evaluated by performing one-sample t-tests in SPM8. Preprocessed gray matter images were included as the main variables of interest, and the various adaptation performance measures were included as covariates. Voxels with an intensity value of less than 0.1 were excluded from the analysis to account for edge effects. Statistical 
significance was determined by using a cluster-level FDR $p<0.05$ to correct for multiple comparisons.

\section{Results}

\section{Behavioral results}

For the manual adaptation task, the repeated measures ANOVA on DE yielded a significant main effect of Block, $F(11,209)=122.89, p<.001, \eta_{p}{ }^{2}=.87$. As Figure $3 \mathrm{a}$ and Table 2 demonstrate, performance decreased when the rotated feedback was introduced in block A1, and participants gradually adapted to this rotated feedback across the following blocks. When the rotated feedback was removed in block B3, participants had to re-adapt to the normal feedback. The results also yielded a significant Block $\mathrm{x}$ Trial interaction, $F(165,3135)=2.19, p<.01, \eta_{p}{ }^{2}=.10$. Post-hoc analyses revealed that DE significantly decreased across trials in blocks A1-B3, $F_{s}>1.82, p s<.05, \eta_{p}{ }^{2} s>.088$, indicating within-block performance improvements. Additionally, the results revealed a significant Session x Block interaction, $F(11,209)=4.91, p<.001, \eta_{p}{ }^{2}=.21$. Post-hoc analyses demonstrated that DE differed significantly across sessions 1 and 2 in blocks A1-A3, $F s>9.51, p s<.01, \eta_{p}{ }^{2}>.33$, supporting that savings occurred from session 1 to session 2 .

For RT, results revealed a significant main effect of Block, $F(11$, $209)=11.22, p<.001, \eta_{p}{ }^{2}=.37$, as well as a significant Block $\mathrm{x}$ Trial interaction, $F(165$, $3135)=1.56, p<.01, \eta_{p}{ }^{2}=.076$ (see Figure $3 \mathrm{~b}$ and Table 2). However, this interaction was 
no longer significant after excluding the first block from the analysis $(p=.08)$, indicating that RTs decreased more quickly in the first block of the adaptation task than in the remaining blocks. Results also showed a significant Session $\mathrm{x}$ Trial interaction, $F(15$, $285)=3.70, p<.001, \eta_{p}{ }^{2}=.16$. However, this interaction was no longer significant after excluding the first trial from each block $(p=.23)$.

The results for MT yielded significant main effects of Session, $F(1$, $19)=27.90, p<.001, \eta_{p}{ }^{2}=.60$, Block, $F(11,209)=35.21, p<.001, \eta_{p}{ }^{2}=.65$, and Trial, $F(15$, $285)=12.29, p<.001, \eta_{p}{ }^{2}=.39$ (see Figure $3 c$ and Table 2 ). Additionally, there was a significant Session $\mathrm{x}$ Block interaction, $F(11,209)=9.08, p<.001, \eta_{p}{ }^{2}=.32$. Post-hoc analyses demonstrated that MT differed significantly between sessions 1 and 2 within blocks B1-A3, and A5 and A7, Fs>5.67, $p s<.05, \eta_{p}{ }^{2}>.23$, suggesting that savings across the two sessions is also reflected in MT in addition to DE.

To examine how well participants remembered the adaptation task from session 1 to session 2, we calculated individual saving scores. This score was defined as the difference between a participant's mean DE in the first adaptation block (i.e., the first 16 trials with rotated feedback) on the first session and the mean DE in that block on the second session. Savings scores ranged from -35.58 to 6.53 degrees, with more negative scores reflecting more savings. To determine whether saving scores were significantly different from zero, we performed a one-sample t-test. The results showed that overall participants had significant savings, with a mean score of $-8.73, t(19)=-3.69, p<.01$. Our individual differences measure of savings could comprise both retention and savings effects. To investigate this, we performed a linear contrast to evaluate whether performance changes across trials in block $A 1$ were different for the two test 
sessions. As illustrated in Figure 3d, results revealed that DE improved significantly faster across trials in the second compared to the first test session, $F(1,19)=5.26$, $p<.05, \eta_{p}{ }^{2}=.22$. It is clear from the figure that performance is essentially identical across the first few trials of block $\mathrm{A} 1$ for both Sessions 1 and 2, supporting that the session difference in A1 average direction error is based on savings (rate of adaptation) and not retention. However, it is difficult to disentangle the effects of retention from savings; this issue should thus be investigated in future studies.

\section{(Enter Figure 3 and Table 2 here)}

\section{Functional connectivity results}

For the seed-to-voxel analyses, results revealed that functional connectivity among the left dorsal anterior cingulate cortex (dACC) seed region with supplementary motor area (SMA), bilateral insula, putamen and thalamus and left cerebellar lobule V was predictive of the rate of early adaptation, such that faster learners exhibited greater connectivity strength within this network. In addition, connectivity between left posterior cingulate cortex seed region with precuneus cortex, bilateral occipital cortices, and superior frontal gyrus (comprising the default mode network; DMN) was associated with the rate of late adaptation and savings scores. Specifically, we observed that DMN connectivity was stronger in participants who adapted faster during the late phase of the visuomotor adaptation task and for those who showed better savings in the second test session (see Figures 4 and 5). The DMN is a set of brain areas that exhibit higher 
metabolic activity at rest than during performance of externally-directed tasks. Important functions attributed to this network are the support of internally directed mental activity (Gusnard et al. 2001; Mason et al. 2007) and memory consolidation (Miall and Robertson 2006).

(Enter Figures 4 and 5 here)

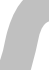

Voxel-to-voxel analyses also revealed significant associations between the rate of learning in the early adaptation phase as well as aftereffects with functional connectivity. Specifically, left insular cortex connectivity was correlated with the rate of early adaptation, such that faster learners exhibited greater connectivity strength between left insular cortex with the rest of the brain. Furthermore, we found that connectivity strength between right operculum cortex with the rest of the brain was stronger in individuals who adapted faster to the removal of rotated feedback during the post adaptation blocks (see Table 2 and Figure 6). Thus, for both seed-to-voxel and voxel-to-voxel approaches, stronger functional connectivity was always associated with better performance.

(Enter Table 2 and Figure 6 here)

\section{Voxel-based morphometry results}

The VBM results showed significant correlations between the rates of early and late adaptation and gray matter volume. Specifically, participants who had faster rates of adaptation during the early phase of the visuomotor adaptation task showed larger gray 
matter volume in left central operculum cortex, precuneus cortex, and left postcentral gyrus. In contrast, participants who adapted faster during the late phase of the adaptation task had larger gray matter volume in a cluster including vermis VI and right crus I of the cerebellum (see Table 3 and Figure 7). There was no association between gray matter volume and aftereffects or savings. Thus, larger gray matter volume was always associated with better performance.

(Enter Table 3 and Figure 7 here)

Relationship between connectivity strength and GM volume

In order to explore the relationship between GM volume and fcMRI in the overlapping brain regions that showed associations with behavior, we first correlated the neural measures (fcMRI and GM volume) in left temporoparietal cortex (region associated with early adaptation). We found a significant positive relationship between GM volume and fcMRI in this region $(r=0.71 ; p<0.0001)$. Next, we correlated each participant's average DMN connectivity measure and GM volume in Vermis VI of the cerebellum (regions associated with late adaptation), but this relationship was not significant $(r=-0.20 ; p=.26)$.

In order to more fully investigate the potential role of fcMRI as a mechanistic link between GM volume and the rate of early/late adaptation, we applied mediation analyses to the VBM, fcMRI, and behavioral data. Mediation analyses examine the mechanism by which two variables are related, and is 
appropriate when the mediator $(M)$ is the logical effect of one variable $(X)$, and the logical cause of another variable (Y). In this study, fcMRI is the logical effect of GM volume $(\mathrm{X})$ and the logical cause of differences in adaptation rate (Y).

We performed a first mediation analysis using GM volume is temporoparietal cortex as the independent variable $(\mathrm{X})$, fcMRI in temporoparietal cortex as the mediator $(M)$ and early adaptation rate as the outcome variable (Y). In step 1 of the mediation model, the regression of GM volume on early adaptability, ignoring the mediator (fcMRI) was significant, $b=-.0001, t(32)=-4.09, p<.001$. Step 2 showed that the regression of GM volume on the mediator, fcMRI, was also significant, $b=.0032, t(32)=5.69, p<.0001$. Step 3 of the mediation process showed that the mediator (fcMRI), controlling for GM volume, was not significant, $b=-.02$, $t(31)=-1.91, p=.07$. Step 4 of the analyses revealed that, controlling for the mediator (fcMRI), GM volume was not a significant predictor of early adaptability, $b=-.0001$, $t(31)=-1.65, p=.11$. A Sobel test was then conducted which yielded a non-significant mediation in the model $(\mathrm{z}=-\mathbf{1 . 7 8}, p=.07)$. Thus, fcMRI did not fully mediate the relationship between GM volume and early adaptability. We performed a second mediation analysis using GM volume in Vermis VI of the cerebellum as the independent variable $(\mathrm{X})$, fcMRI in DMN as the mediator (M) and late adaptation rate as the outcome variable (Y). Here, too, results showed that the regression of GM volume on late adaptability, ignoring the mediator (fcMRI) was significant, $b=-.001, t(32)=-3.93, p<.001$, while the regression of $G M$ volume on fcMRI was not significant, $b=-.0002, t(32)=-1.16, p=.26$. Step 3 of the mediation process showed that the mediator (fcMRI), controlling for GM volume, 
was significant, $b=-.84, t(31)=-4.17, p<.001$. Step 4 of the analyses revealed that, controlling for the mediator (fcMRI), GM volume was a significant predictor of late adaptability, $b=-.0013, t(31)=-5.57, p<.0001$. A Sobel test was then conducted which yielded a non-significant mediation in the model $(z=1.09, p=.28)$. Thus, like above this, analysis showed no indications that fcMRI fully mediated the relationship between GM volume and late adaptability.

\section{Discussion}

In the present study we evaluated whether individual differences in the rate of visuomotor adaptation and savings magnitude are associated with differences in offline measures of brain structural and functional properties. We observed that specific patterns of resting state functional connectivity strength and gray matter volume were associated with individual variability in learning rates and multi-day savings, suggesting that these patterns represent neural predictors of sensorimotor adaptability and savings. Below, we will first elaborate on our findings regarding predictors of learning rate and then discuss our findings related to multi-day savings in sensorimotor adaptation.

\section{Predictors of adaptability}

1

While previous investigations have reported associations between the rate of learning during the early adaptation phase and brain structural and online functional measures, the present study is the first to demonstrate that resting state functional connectivity strength and gray matter volume are associated with the rate of learning 
during different phases of visuomotor adaptation. For the early phase of adaptation which we defined here as the first 64 trials with rotated feedback - faster adapters exhibited stronger functional connectivity between dACC, SMA, bilateral insula, putamen and thalamus and left cerebellar lobule V. In terms of structural predictors, faster adapters exhibited larger gray matter volume in temporoparietal and occipital regions, partially overlapping with findings from the connectivity analyses. For the late adaptation phase - i.e., the final 64 trials with rotated feedback - faster adapters showed greater connectivity strength between DMN regions and larger gray matter volume in superior posterior regions of the cerebellum. These findings support the existence of differential learning processes contributing to sensorimotor adaptation (Smith et al. 2006; Heuer et al. 2015), with fast explicit, strategic and slower implicit, automated processes jointly contributing to performance throughout the task but dominating at different time courses (Taylor et al. 2014).

Consistent with our hypotheses, participants with stronger resting state functional connectivity between dACC seed region with SMA, insula, putamen, thalamus and cerebellum adapted faster during the early phase of the adaptation task. Neuroimaging studies suggest that the dACC plays a critical role in the cognitive aspect of movement generation, i.e., intentional motor control (Hoffstaedter et al. 2013, 2014). In particular, this region is known to be involved in evaluating and assigning values to actions based on relevant feedback (Ridderinkhof et al. 2004; Ullsperger and von Cramon 2003). According to this view, successful movements (i.e., those that are correct for counteracting the perturbation in a sensorimotor adaptation task) get assigned higher value and thus are more likely to be repeated than unsuccessful ones. 
In addition, our previous work has found an association between functional activity in the dorsal ACC and conditions of high task difficulty, in which motor errors were relatively large and movements were slow (Seidler et al. 2004). This suggests that participants who are better at error detection and correction may garner their advantage at skill learning by utilizing the dACC network during the early phase of adaptation.

Functional neuroimaging evidence links the $\mathrm{dACC}$ with prefrontal, premotor, parietal and insular regions as well as the basal ganglia, thalamus, and cerebellum forming a core network for the internal generation of movement (Hoffstaedter et al. 2014). One interpretation of our results is that high functional connectivity at rest within this network may facilitate the early, more cognitively demanding phase of sensorimotor adaptation.

Also consistent with our hypotheses, faster adapters during the early phase of the task showed greater functional connectivity strength between left insular cortex with the rest of the brain. Furthermore, connectivity strength between right insular cortex with the rest of the brain was stronger in participants who re-adapted to the normal feedback faster. The insular cortex plays an important role in the integration of multimodal sensorimotor and cognitive functions such as error detection, salience, attention to behaviorally relevant stimuli, anticipation, and decision making (Taylor et al., 2009). Previous studies using resting state $\mathrm{fMRI}$ have identified this region as part of an intrinsic task-positive network or "salience network" along with other areas such as DLPFC, SMA, inferior parietal sulcus and frontal eye fields (Fox et al., 2005; Taylor et al., 2009). This intrinsic network could thus be a general salience and action system involved in environmental monitoring, response selection, and other attentional processes required during visuomotor adaptation. 
Interestingly, we observed converging results between functional connectivity and gray matter volume as neural predictors of sensorimotor adaptation. Both analyses indicated a prominent role of left temporoparietal regions in explaining individual variation in early adaptability to the visuomotor task. These findings are in line with previous studies that have explored the neural correlates of sensorimotor adaptation. For instance, Mutha et al. (2013) reported that left parietal regions are crucial for adaptive visuomotor control. They proposed that this region is important for forming and maintaining internal representations of the relationship between motor commands and limb and environmental state. Additionally, Danckert et al. (2008) used event-related fMRI to examine the dynamic effects of prisms lenses on manual pointing. Their results demonstrated that activity in anterior cingulate and intraparietal regions were higher during early compared to late adaptation, suggesting that an extensive network of cingulate and temporoparietal regions is involved in recalibrating visuomotor commands in the face of perturbed visual input.

Another converging finding from multiple imaging modalities within this study was the role of the posterior region of the cerebellum in explaining individual variability in late adaptation. According to the functional network parcellation of the cerebellum, this region is located within the DMN area of the cerebellum (Buckner 2012). Results from both our functional connectivity and VBM analyses indicate that this network can be considered a neural predictor of late adaptation. Overall, these results demonstrate that both temporoparietal and cerebellar regions are associated with faster initial adaptation, whereas only the cerebellum is associated with faster adaptation during the late phase. Results of our mediation analyses showed no significant indications that gray volume and 
connectivity strength jointly contribute to individual differences in adaptability. Future studies should aim to investigate the relationship between measures from multiple imaging modalities and sensorimotor adaptability and savings.

\section{Predictors of savings}

We found behavioral evidence for multi-day savings in sensorimotor adaptation from the first to the second test session. Participants were less perturbed when the rotated feedback was introduced during the second test session compared to the first, indicating that they showed savings of what they learned during their previous experience with the task. At the neural level, we observed that the amount of savings was associated with the degree of resting state functional connectivity among default mode regions of the brain. Specifically, stronger DMN connectivity was associated with more savings. Consistent with some previous studies, these results also demonstrate that the neural correlates associated with savings are similar to those for late learning (Kojima et al. 2004; Medina et al. 2001; Smith et al. 2006; Seidler and Noll 2008). For instance, Seidler and Noll (2008) found that better generalization of adaptation was correlated with higher activity in brain regions that play a role in late adaptation, including the superior posterior fissure of the cerebellum. These findings suggest that savings is less cognitively demanding than acquisition, and further, that stronger DMN resting state connectivity may facilitate the later, more procedural stages of sensorimotor adaptation as well as better savings of the task. 
Although we found evidence for overlapping neural processes between late adaptation and savings, there are reports in the literature supporting that savings is more associated with early learning cognitive / strategic processes (Haith et al. 2015; Morehead et al. 2015; Seidler et al. 2017). One potential explanation for these differential findings is that these previous studies were based on behavioral measures of adaptation (Morehead et al. 2015; Haith et al. 2015; Seidler et al. 2017). In contrast, the present study examined offline neural predictors of performance, or the baseline state that participants bring to the adaptation process. In particular, offline neural predictors reveal individual differences in system configuration that are more likely to facilitate subsequent dynamic states.

A novel and somewhat unexpected finding of the current study is the association between DMN connectivity strength with learning rate during the late adaptation phase and with savings from the first to the second test session. The DMN consists of a set of brain areas that show highly correlated activity and high metabolic demands at rest (Raichle et al. 2001). An important function of this network is to support internally directed mental activity (Gusnard et al. 2001; Mason et al. 2007). The DMN may also make a crucial contribution to the offline processing and consolidation of memories (Miall and Robertson 2006). Memory consolidation can be defined behaviorally as performance becoming less susceptible to interference (Brashers-Krug et al. 1996) or as performance that improves over time in the absence of further practice (Robertson et al. 2004a; Robertson et al. 2004b). Thus, the DMN may play a role in the reprocessing of past experiences to support memory consolidation during the late phase of sensorimotor adaptation, as well as savings of the task. The DMN is considered to be anti-correlated with brain regions involved in cognitive control (Seely et 
al. 2007) such as the dACC network, which we found to be associated with early adaptation. The dynamic opposition between these two networks may therefore be important for regulating attentional and goal-directed demands, and potentially coordinating the interplay between internally and externally directed thought (Raichle 2010). Our results demonstrate that DMN functional connectivity strength is a predictor of the later, more procedural stages of sensorimotor adaptation and savings, potentially allowing better consolidation of the adapted state.

\section{Limitations}

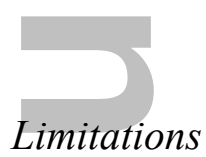

(

One limitation of the present study is that neuroimaging data were collected on different scanners at different sites (i.e., different scanner sequences and staff), which may have affected the results. However, we used scanner site as a nuisance covariate in all analyses to control for this potential confound. Another limitation is that in contrast to the voxel-to-voxel functional connectivity analyses, seed-to-voxel analyses were not corrected for the number of correlations performed, and therefore should be interpreted with caution. However, the magnitude of these correlations is still moderate and therefore provides useful information about the neural predictors of sensorimotor adaptability and savings. It should further be noted that the subject-averaged mask created during the seed-to-voxel analyses was corrected for multiple comparisons.

Lastly, the present study is limited by the unequal distribution of males and females (30 males; 4 females). Men and women differ in many sensory systems, neural anatomy, and functional responses (Mark et al., 2014). For instance, Moreno-Briseño et 
al. (2010) investigated gender differences in a prism adaptation throwing task. They found that males had significantly higher throwing accuracies, although there were no adaptation differences between genders. In contrast, females showed significantly larger negative aftereffects, which could be explained by a larger contribution of spatial alignment. The unequal gender distribution in the present study may thus have prevented us from identifying additional brain-behavior associations. Using gender as a covariate yielded very similar results for our brain-behavior analyses; however, the association between GM volume in the cerebellum and late adaptation was no longer significant $(p=0.12)$ and the association between voxel-to-voxel functional connectivity in right central operculum cortex and the behavioral aftereffects was no longer significant $(p=0.07)$. With our current sample size it is difficult to say whether this should be interpreted as evidence for gender differences in adaptability, or simply as a reduction of power due to the addition of gender as a covariate in the model. Future studies should aim to include equal distributions of males and females in order to test this hypothesis and to be more representative of the population.

\section{Conclusion}

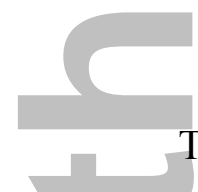

To summarize, we found that functional connectivity strength within a salience network is predictive of the rate of learning during the early phase of a sensorimotor adaptation task. In contrast, DMN functional connectivity strength predicts late learning and multi-day savings, such that faster learners during the late phase and individuals with better savings of the task showed greater connectivity strength within this network. 
In terms of structural predictors, gray matter volume in temporoparietal regions was correlated with the rate of early adaptation, whereas gray matter volume in superior posterior cerebellum was associated with the rate of learning during the late phase of the

visuomotor task. We propose that the functional and structural correlates of early adaptation likely support cognitive components of the visuomotor task, such as working memory, error detection and correction, and attention. In contrast, the neural correlates of late performance likely support the storage and modification of newly acquired sensorimotor representations.

Better multi-day savings was associated with stronger resting state functional connectivity in the same network involved in late adaptation, suggesting overlapping neural processes between these two phases of sensorimotor adaptation. Ultimately, being able to identify people who are slower adapters could have important implications for developing targeted training programs that enhance adaptation learning, for example with respect to spaceflight (Bloomberg et al., 2015; Seidler et al., 2015) or rehabilitation (Bastian, 2008).

\section{Acknowledgements}

This work was supported by grants from the National Space Biomedical Research Institute (NASA NCC 9-58, MA02701, and PF04101), from the National Aeronautics and Space Administration (NASA; NNX11AR02G) and NASA Flight Analogs Project, and the National Institutes of Health, and National Center for Advancing Translational Sciences, 1UL1RR029876-01 


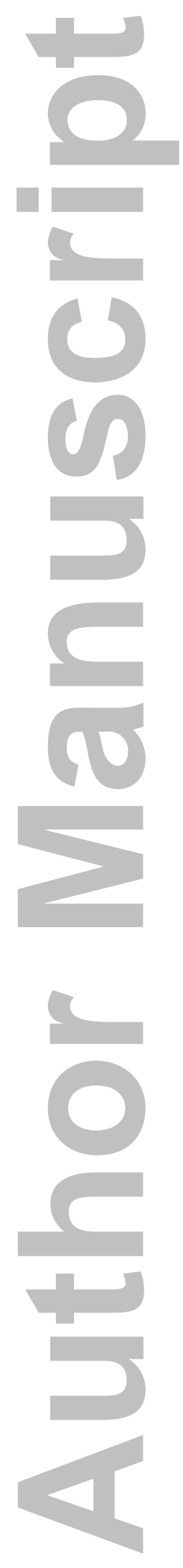

John Wiley \& Sons, Inc.

This article is protected by copyright. All rights reserved. 


\section{References}

Refert

C

Albert NB, Robertson EM, Miall RC (2009): The resting human brain and motor

learning. Current Biology 19:1023-1027.

Anguera JA, Reuter-Lorenz PA, Willingham DT, Seidler RD (2010): Contributions of spatial working memory to visuomotor learning. Journal of Cognitive

Neuroscience 22:1917-1930.

Anguera JA, Reuter-Lorenz PA, Willingham, DT, Seidler RD (2011): Failure to engage spatial working memory contributes to age-related declines in visuomotor

learning. Journal of Cognitive Neuroscience 23:11-25.

Anguera JA, Russell CA, Noll DC, Seidler RD (2007): Neural correlates associated with intermanual transfer of sensorimotor adaptation. Brain Research 1185:136-151.

Ashburner J, Friston KJ (2005). Unified segmentation. Neuroimage, 26:839-851.

Avants BB, Tustison NJ, Song G, Cook PA, Klein A, Gee JC (2011): A reproducible evaluation of ANTs similarity metric performance in brain image

registration. Neuroimage 54:2033-2044.

John Wiley \& Sons, Inc. 
Bastian AJ (2008). Understanding sensorimotor adaptation and learning for rehabilitation. Current opinion in neurology, 21:628.

Bédard P, Sanes JN (2011). Basal ganglia-dependent processes in recalling learned visual-motor adaptations. Experimental brain research, 209:385-393.

Behzadi Y, Restom K, Liau J, Liu TT (2007): A component based noise correction method (CompCor) for BOLD and perfusion based fMRI. Neuroimage 37:90-101.

Bloomberg JJ, Peters BT, Cohen HS, Mulavara AP (2015). Enhancing astronaut performance using sensorimotor adaptability training. Frontiers in systems neuroscience, 9.

Bock O (1992): Adaptation of aimed arm movements to sensorimotor discordance: evidence for direction-independent gain control. Behavioural Brain Research 51:41-50.

Bock O, Burghoff M (1997): Visuo-motor adaptation: evidence for a distributed amplitude control system. Behavioural Brain Research 89:267-273.

Brashers-Krug T, Shadmehr R, Bizzi E (1996). Consolidation in human motor memory. Nature, 382:252. 
the human cerebellum estimated by intrinsic functional connectivity. Journal of Neurophysiology 106:2232-2245.

Clower DM, Hoffman JM, Votaw JR, Faber TL, Woods RP, Alexander GE (1996): Role of posterior parietal cortex in the recalibration of visually guided reaching. Nature $383: 618-621$.

\section{(1)}

Danckert J, Ferber S, Goodale MA (2008): Direct effects of prismatic lenses on visuomotor control: an event $\square$ related functional MRI study. European Journal of Neuroscience 28:1696-1704.

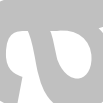

Deiber MP, Honda M, Ibañez V, Sadato N, Hallett M (1999): Mesial motor areas in selfinitiated versus externally triggered movements examined with fMRI: effect of movement type and rate. Journal of Neurophysiology 81:3065-3077.

Della-Maggiore V, Landi SM, Villalta JI (2015): Sensorimotor Adaptation Multiple Forms of Plasticity in Motor Circuits. The Neuroscientist 21:109-125.

Della-Maggiore V, McIntosh AR (2005). Time course of changes in brain activity and functional connectivity associated with long-term adaptation to a rotational transformation. Journal of neurophysiology, 93:2254-2262. 
Della $\square$ Maggiore V, Scholz J, Johansen $\square$ Berg H, Paus T (2009): The rate of visuomotor adaptation correlates with cerebellar white $\square$ matter microstructure. Human Brain Mapping 30:4048-4053.

Diedrichsen J (2006): A spatially unbiased atlas template of the human cerebellum. Neuroimage 33:127-138.

Flament D, Ellermann JM, Kim SG, Uğurbil K, Ebner TJ (1996). Functional magnetic resonance imaging of cerebellar activation during the learning of a visuomotor dissociation task. Human Brain Mapping 4:210-226.

Fox MD, Snyder AZ, Vincent JL, Corbetta M, Van Essen DC, Raichle ME (2005). The human brain is intrinsically organized into dynamic, anticorrelated functional networks. Proceedings of the National Academy of Sciences of the United States of America, $102: 9673-9678$.

Ghilardi MF, Ghez C, Dhawan V, Moeller J, Mentis M, Nakamura T, et al (2000):

Patterns of regional brain activation associated with different forms of motor

learning. Brain Research 871:127-145.

$1-$

Gusnard DA, Raichle ME (2001): Searching for a baseline: functional imaging and the resting human brain. Nature Reviews Neuroscience 2:685-694. 
Haith AM, Huberdeau DM, Krakauer JW (2015): The influence of movement preparation time on the expression of visuomotor learning and savings. The Journal of Neuroscience 35:5109-5117.

Heuer H, Hegele M (2015): Explicit and implicit components of visuo-motor adaptation: An analysis of individual differences. Consciousness and Cognition 33:156-169.

Hoffstaedter F, Grefkes C, Zilles K, Eickhoff SB (2013): The "what" and "when” of selfinitiated movements. Cerebral Cortex 23:520-530.

Hoffstaedter F, Grefkes C, Caspers S, Roski C, Palomero $\square$ Gallagher N, Laird AR, et al. (2014): The role of anterior midcingulate cortex in cognitive motor control. Human Brain Mapping 35:2741-2753.

Hutton C, Draganski B, Ashburner J, Weiskopf N (2009). A comparison between voxelbased cortical thickness and voxel-based morphometry in normal aging. Neuroimage 48:371-380.

Huynh H, Feldt LS (1970): Conditions under which mean square ratios in repeated measurements designs have exact F-distributions. Journal of the American Statistical Association 65:1582-1589. 
Imamizu H, Miyauchi S, Tamada T, Sasaki Y, Takino R, PuÈtz B, et al. (2000): Human cerebellar activity reflecting an acquired internal model of a new tool. Nature 403:192195.

Inoue K, Kawashima R, Satoh K, Kinomura S, Goto R, Sugiura M, et al. (1997): Activity in the parietal area during visuomotor learning with optical rotation. Neuroreport 8:39793983.

Kojima Y, Iwamoto Y, Yoshida K (2004). Memory of learning facilitates saccadic adaptation in the monkey. Journal of Neuroscience 24:7531-7539.

Krakauer JW, Pine ZM, Ghilardi MF, Ghez C (2000): Learning of visuomotor transformations for vectorial planning of reaching trajectories. The Journal of Neuroscience 20:8916-8924.

Landi SM, Baguear F, Della-Maggiore V (2011): One week of motor adaptation induces structural changes in primary motor cortex that predict long-term memory one year later. The Journal of Neuroscience 31:11808-11813.

Mark S, Scott GB, Donoviel DB, Leveton LB, Mahoney E, Charles JB, Siegel B (2014). The impact of sex and gender on adaptation to space: executive summary. Journal of Women's Health 23:941-947. 
Martuzzi R, Ramani R, Qiu M, Shen X, Papademetris X, et al. (2011): A whole-brain voxel based measure of intrinsic connectivity contrast reveals local changes in tissue connectivity with anesthetic without a priori assumptions on thresholds or regions of interest. Neuroimage 58:1044-1050.

Mason MF, Norton MI, Van Horn JD, Wegner DM, Grafton ST, Macrae CN (2007):

Wandering minds: the default network and stimulus-independent

thought. Science 315:393-395.

Medina JF, Garcia KS, Mauk MD (2001). A mechanism for savings in the cerebellum. Journal of Neuroscience 21:4081-4089.

Morehead JR, Qasim SE, Crossley MJ, Ivry R (2015): Savings upon re-aiming in visuomotor adaptation. The Journal of Neuroscience 35:14386-14396.

Moreno-Briseño P, Díaz R, Campos-Romo A, Fernandez-Ruiz J (2010). Sex-related differences in motor learning and performance. Behavioral and brain functions 6:74.

Mutha PK, Sainburg RL, Haaland KY (2011): Left parietal regions are critical for adaptive visuomotor control. The Journal of Neuroscience 31:6972-6981.

Nasir SM, Darainy M, Ostry DJ (2013): Sensorimotor adaptation changes the neural coding of somatosensory stimuli. Journal of Neurophysiology 109:2077-2085. 
Pine ZM, Krakauer JW, Gordon J, Ghez C (1996): Learning of scaling factors and reference axes for reaching movements. Neuroreport 7:2357-2362.

Raichle ME (2010): Two views of brain function. Trends in Cognitive Sciences 14:180190.

Raichle ME, MacLeod AM, Snyder AZ, Powers WJ, Gusnard DA, Shulman GL (2001):

A default mode of brain function. Proceedings of the National Academy of

Sciences 98:676-682.

Robertson

Robertson EM, Pascual-Leone A, Press DZ (2004). Awareness modifies the skilllearning benefits of sleep. Current Biology 14:208-212.

Robertson EM, Pascual-Leone A, Miall RC (2004). Current concepts in procedural consolidation. Nature reviews. Neuroscience 5:576-582.

Ruitenberg MFL, De Dios YE, Gadd NE, Wood SJ, Reuter-Lorenz PA, Kofman I, ... \& Seidler RD (2017). Multi-day Adaptation and Savings in Manual and Locomotor Tasks. Journal of Motor Behavior. Epub ahead of print. Doi: 10.1080/00222895.2017.1371110 Scanlon C, Mueller SG, Tosun D, Cheong I, Garcia P, Barakos J, ... \& Laxer KD (2011). Impact of methodologic choice for automatic detection of different aspects of brain 
atrophy by using temporal lobe epilepsy as a model. American Journal of Neuroradiology

\section{$32: 1669-1676$.}

Seeley WW, Menon V, Schatzberg AF, Keller J, Glover GH, Kenna H, et al. (2007):

Dissociable intrinsic connectivity networks for salience processing and executive control. The Journal of Neuroscience 27:2349-2356.

Seidler RD, Mulavara AP, Bloomberg JJ, Peters BT (2015). Individual predictors of sensorimotor adaptability. Frontiers in systems neuroscience 9.

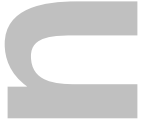

Seidler RD, Gluskin BS, Greeley B (2017). Right prefrontal cortex transcranial direct current stimulation enhances multi-day savings in sensorimotor adaptation. Journal of neurophysiology 117:429-435.

Seidler RD, Noll DC (2008): Neuroanatomical correlates of motor acquisition and motor transfer. Journal of Neurophysiology 99:1836-1845.

Seidler RD, Noll DC, Chintalapati P (2006): Bilateral basal ganglia activation associated with sensorimotor adaptation. Experimental Brain Research 175:544-555.

-

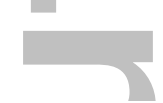

Seidler RD, Noll DC, Thiers G (2004): Feedforward and feedback processes in motor control. Neuroimage 22:1775-1783. 
Shackman AJ, Salomons TV, Slagter HA, Fox AS, Winter JJ, et al. (2011): The

integration of negative affect, pain and cognitive control in the cingulate cortex. Nature

Reviews Neuroscience 12:154-167.

Shadmehr R, Holcomb HH (1997): Neural correlates of motor memory

consolidation. Science 277:821-825.

Shadmehr R, Mussa-Ivaldi FA (1994): Adaptive representation of dynamics during learning of a motor task. The Journal of Neuroscience 14:3208-3224.

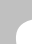

C

Smith MA, Ghazizadeh A, Shadmehr R (2006): Interacting adaptive processes with (r)

different timescales underlie short-term motor learning. PLoS Biology 4:e179.

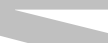

Taylor JA, Krakauer JW, Ivry RB (2014): Explicit and implicit contributions to learning in a sensorimotor adaptation task. The Journal of Neuroscience 34:3023-3032.

Taylor KS, Seminowicz DA, Davis KD (2009). Two systems of resting state connectivity between the insula and cingulate cortex. Human Brain Mapping 30:2731-2745.

Tustison NJ, Avants BB, Cook PA, Zheng Y, Egan A, Yushkevich PA, Gee JC (2010): N4ITK: improved N3 bias correction. IEEE Transactions on Medical Imaging 29:13101320.

John Wiley \& Sons, Inc. 
Vahdat S, Darainy M, Milner TE, Ostry DJ (2011): Functionally specific changes in resting-state sensorimotor networks after motor learning. The Journal of Neuroscience r. 31:16907-16915.

Villalta JI, Landi SM, Fló A, Della-Maggiore V (2013). Extinction interferes with the retrieval of visuomotor memories through a mechanism involving the sensorimotor cortex. Cerebral Cortex 25:1535-1543.

Whitfield-Gabrieli S, Nieto-Castanon A (2012): Conn: a functional connectivity toolbox for correlated and anticorrelated brain networks. Brain Connectivity 2:125-141. 


\section{Figure Legends}

(1)

Figure 1. Experimental design. Participants completed the manual adaptation task while lying supine in the MRI scanner on two separate test sessions (top row). The task consisted of 12 blocks of 16 trials each. B refers to baseline blocks involving normal feedback; A refers to adaptation blocks in which feedback was rotated $45^{\circ}$ clockwise.

Figure 2. Illustration of the visuomotor adaptation task and calculation of direction error (DE). a) DE was calculated as the angle between the line connecting the start and target positions (in joystick coordinates) and the line connecting the start with the spatial location of the joystick at the time of peak velocity. b) Illustration of example cursor movement during early phase of adaptation task. c) Illustration of example cursor movement during late phase of adaptation task.

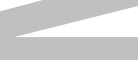

Figure 3. Mean (a) DE, (b) RT, and (c) MT as a function of block within the adaptation task. (d) Mean DE as a function of trial within block A1. Blue lines represent the data from session 1; red lines represent the data from session 2.

Figure 4. Top row: Greater functional connectivity strength among left dACC seed region (in blue) with SMA, bilateral insula, putamen and thalamus, and left cerebellar lobule V was associated with faster early adaptation. Bottom row: Greater functional connectivity strength between left posterior cingulate cortex seed region (in blue) with 
precuneus cortex, bilateral occipital cortices, and superior frontal gyrus (comprising the DMN) was associated with faster late adaptation and more savings.

Figure 5. Scatterplots illustrating the relationship between dACC network functional connectivity strength and early adaptation rate (top row), DMN connectivity strength and late adaptation rate (middle row), and DMN connectivity strength and savings scores (bottom row). Note that more negative values reflect faster adaptation / more savings.

Figure 6. Left insular cortex functional connectivity was correlated with the rate of early adaptation, such that faster learners showed greater connectivity strength between left insular cortex with the rest of the brain than slower learners (top row). Functional connectivity strength between right central operculum cortex with the rest of the brain was stronger in individuals who re-adapted to the visuomotor task faster (bottom row).

Figure 7 Gray matter volume in left central operculum cortex (first row), precuneus cortex (second row), and left postcentral gyrus (third row) was correlated with the rate of early adaptation, in which faster learners exhibited higher gray matter volume in these regions than slower learners. Gray matter volume in vermis VI and right crus I of the cerebellum (fourth row) was higher in participants that showed faster rates of adaptation during the late phase of the task. 


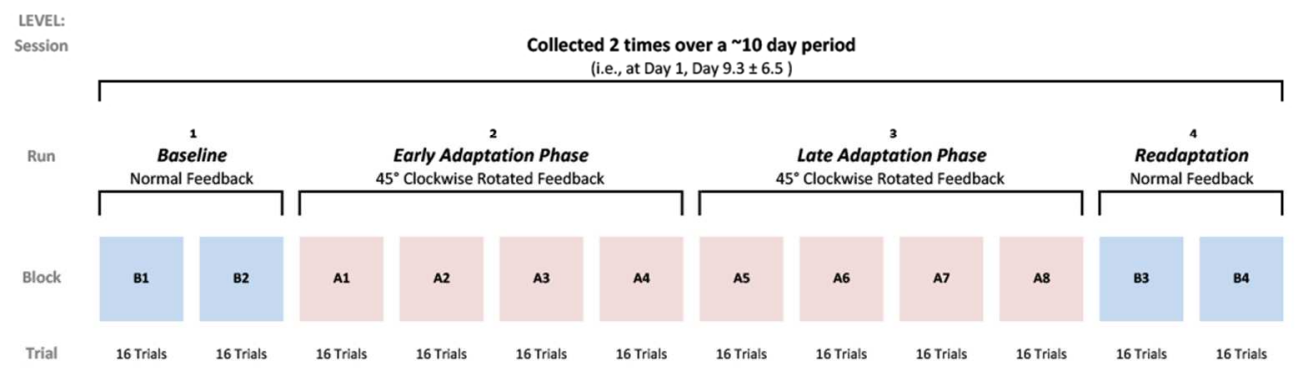

Figure 1. Experimental design. Participants completed the manual adaptation task while lying supine in the MRI scanner on two separate test sessions (top row). The task consisted of 12 blocks of 16 trials each. B refers to baseline blocks involving normal feedback; A refers to adaptation blocks in which feedback was rotated $45^{\circ}$ clockwise.

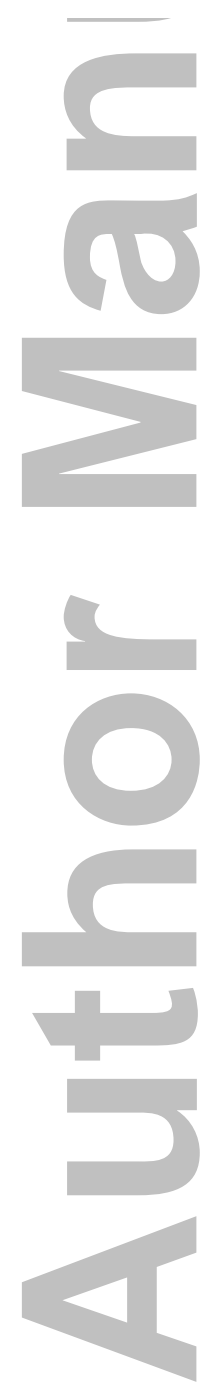

$367 \times 106 \mathrm{~mm}(72 \times 72$ DPI $)$

John Wiley \& Sons, Inc.

This article is protected by copyright. All rights reserved. 


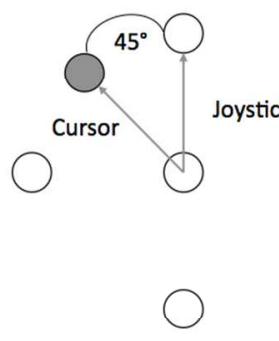

a.

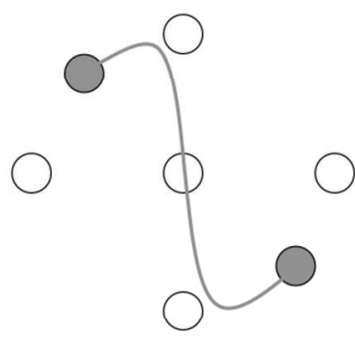

b.

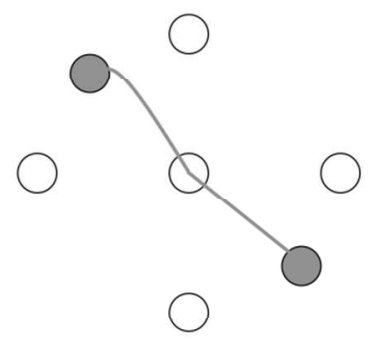

c.

Figure 2. Illustration of the visuomotor adaptation task and calculation of direction error (DE). a) DE was calculated as the angle between the line connecting the start and target positions (in joystick coordinates) and the line connecting the start with the spatial location of the joystick at the time of peak velocity. b) Illustration of example cursor movement during early phase of adaptation task. c) Illustration of example cursor movement during late phase of adaptation task.

$409 \times 150 \mathrm{~mm}(72 \times 72$ DPI $)$
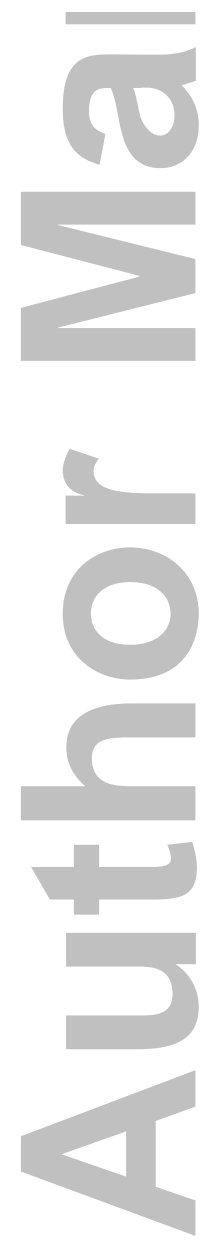

John Wiley \& Sons, Inc. 

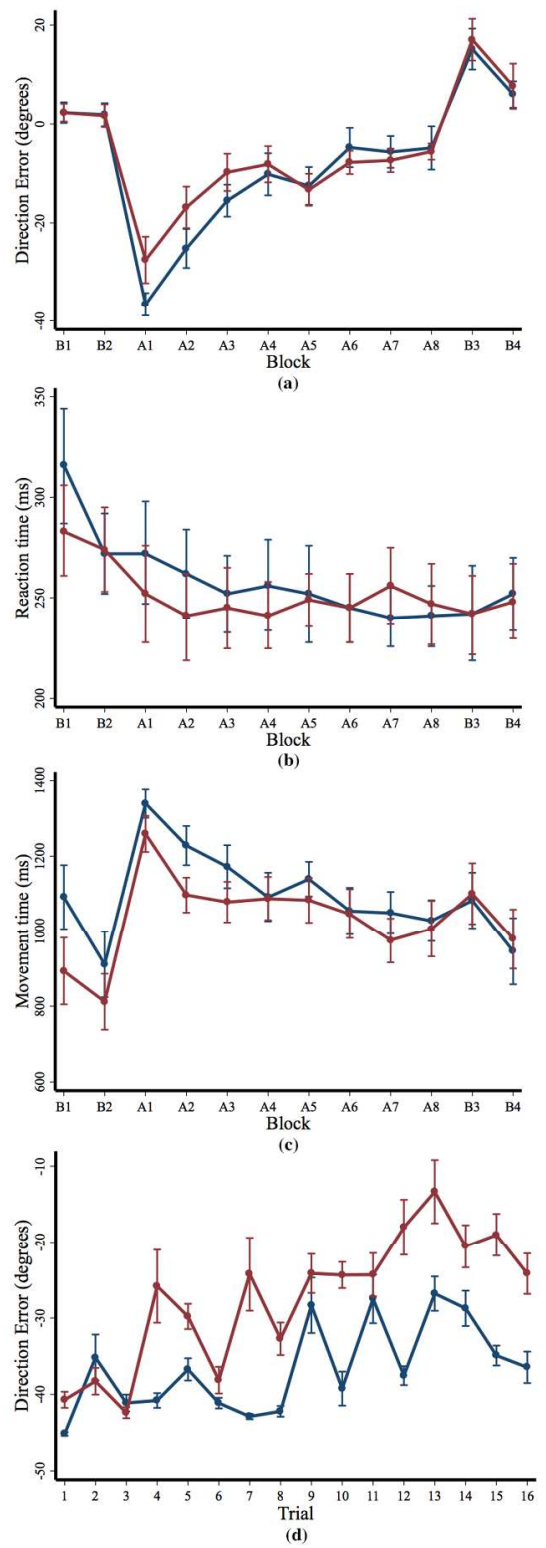

Figure 3. Mean (a) DE, (b) RT, and (c) MT as a function of block within the adaptation task. (d) Mean DE as a function of trial within block $A 1$. Blue lines represent the data from session 1 ; red lines represent the data from session 2 .

$323 \times 896 \mathrm{~mm}(72 \times 72 \mathrm{DPI})$

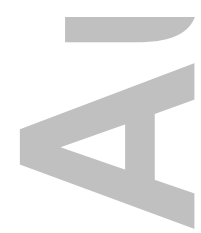

John Wiley \& Sons, Inc.

This article is protected by copyright. All rights reserved. 

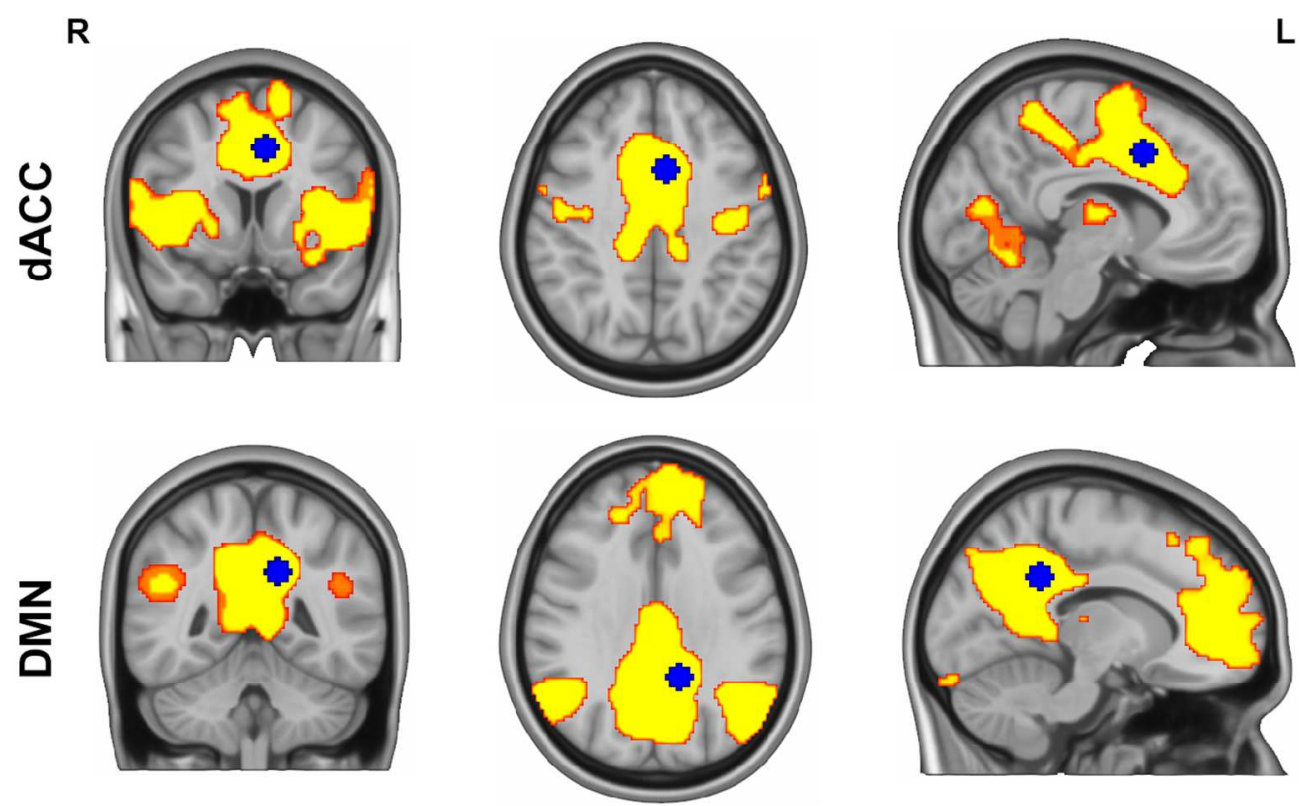

Figure 4. Top row: Greater functional connectivity strength among left dACC seed region (in blue) with SMA, bilateral insula, putamen and thalamus, and left cerebellar lobule $\mathrm{V}$ was associated with faster early adaptation. Bottom row: Greater functional connectivity strength between left posterior cingulate cortex seed region (in blue) with precuneus cortex, bilateral occipital cortices, and superior frontal gyrus (comprising the DMN) was associated with faster late adaptation and more savings.

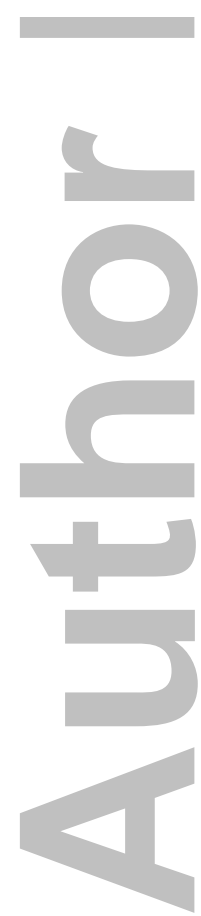

$468 \times 289 \mathrm{~mm}(72 \times 72$ DPI $)$

John Wiley \& Sons, Inc.

This article is protected by copyright. All rights reserved. 

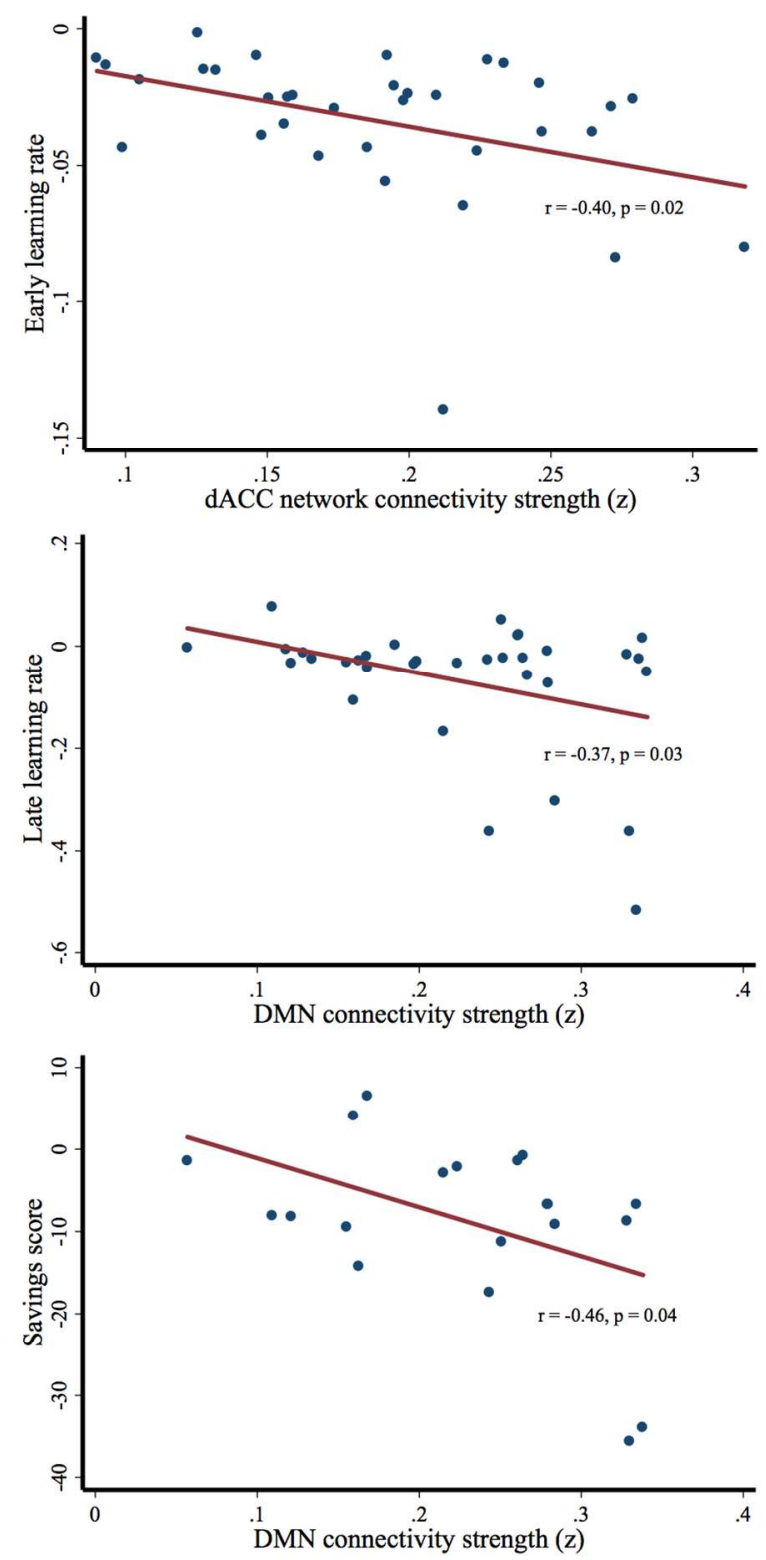

Figure 5. Scatterplots illustrating the relationship between $d \neg A C C$ network functional connectivity strength and early adaptation rate (top row), DMN connectivity strength and late adaptation rate (middle row), and DMN connectivity strength and savings scores (bottom row). Note that more negative values reflect faster adaptation / more savings.

$310 \times 636 \mathrm{~mm}(72 \times 72 \mathrm{DPI})$

John Wiley \& Sons, Inc.

This article is protected by copyright. All rights reserved. 


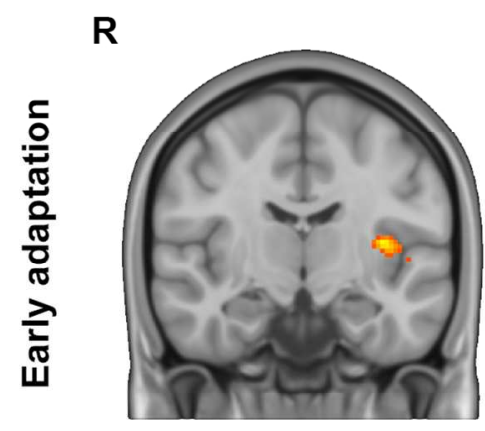

$y=-12$

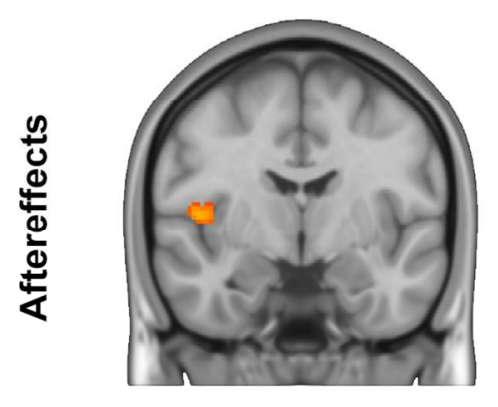

$y=-6$

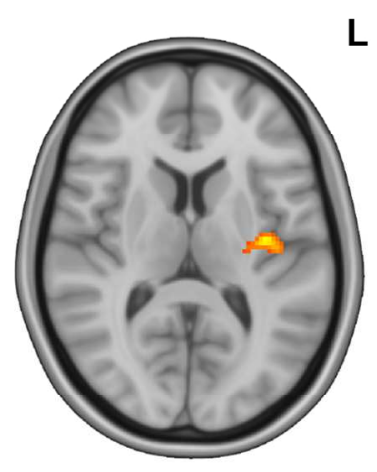

$z=10$

$z=8$

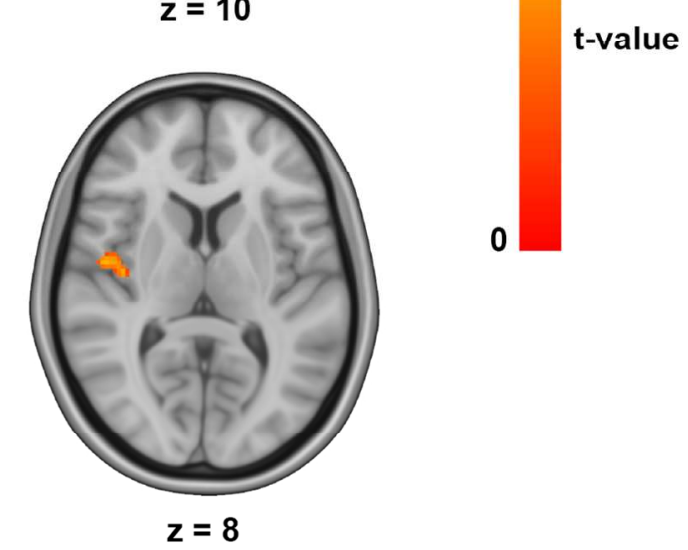

5.21

t-value

Figure 6. Left insular cortex functional connectivity was correlated with the rate of early adaptation, such that faster learners showed greater connectivity strength between left insular cortex with the rest of the brain than slower learners (top row). Functional connectivity strength between right central operculum cortex with the rest of the brain was stronger in individuals who re-adapted to the visuomotor task faster (bottom row).

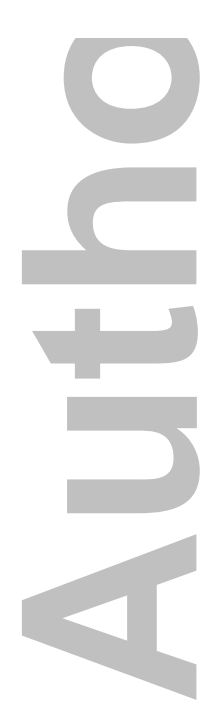

$553 \times 442 \mathrm{~mm}(72 \times 72 \mathrm{DPI})$

John Wiley \& Sons, Inc.

This article is protected by copyright. All rights reserved. 


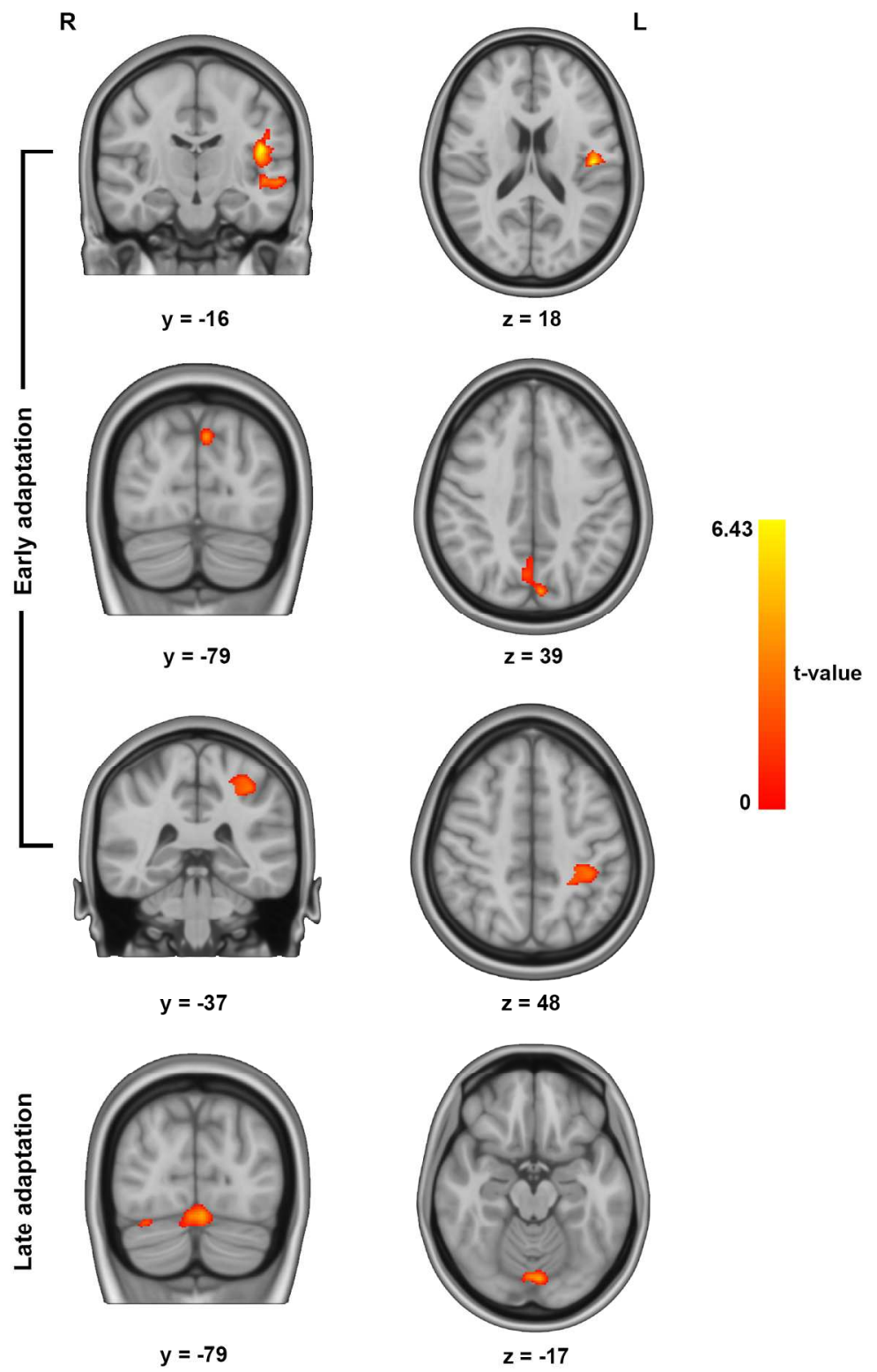

Figure 7 Gray matter volume in left central operculum cortex (first row), precuneus cortex (second row), and left postcentral gyrus (third row) was correlated with the rate of early adaptation, in which faster learners exhibited higher gray matter volume in these regions than slower learners. Gray matter volume in vermis VI and right crus I of the cerebellum (fourth row) was higher in participants that showed faster rates of adaptation during the late phase of the task.

$570 \times 904 \mathrm{~mm}(72 \times 72$ DPI $)$

John Wiley \& Sons, Inc.

This article is protected by copyright. All rights reserved. 
Table I. Peak coordinates of the regions of interest (ROIs) used for seed-to-voxel functional connectivity analyses. Cortical ROIs were defined as $6 \mathrm{~mm}$-radius spheres, and subcortical ROIs were defined as $4 \mathrm{~mm}$-radius spheres around peak coordinates.

\begin{tabular}{lcc}
\hline \hline \multicolumn{1}{c}{ Seed region } & MNI coordinates (x,y,z) & Reference \\
\hline R Middle frontal gyrus & $44,14,36$ & Anguera et al. (2011) \\
R Inferior parietal lobule & $48,-40,44$ & Anguera et al. (2010) \\
L Putamen & $26,6,0$ & Di Martino et al. (2008) \\
L Superior temporal gyrus & $-46,-34,14$ & Anguera et al. (2010) \\
L Middle temporal gyrus & $-48,-48,6$ & Anguera et al. (2010) \\
R Globus pallidus & $20,-6,0$ & Della-Maggiore et al. (2005) \\
L Anterior cingulate cortex & $-7,9,40$ & Seidler and Noll (2008) \\
L Cerebellum lobule V & $-11,-56,-13$ & Seidler and Noll (2008) \\
L Cerebellum lobule VI & $-26,-62,-20$ & Seidler and Noll (2008) \\
L Posterior cingulate cortex & $-12,-47,32$ & Greicius et al. (2003) \\
\hline \hline
\end{tabular}

John Wiley \& Sons, Inc. 
Table II. Mean and standard error of the mean (SEM) for direction error (DE), movement time (MT) and reaction time (RT) per block during Sessions 1 and 2.

\begin{tabular}{|c|c|c|c|c|c|c|c|}
\hline Session & Block & DE Mean & DE SEM & MT Mean & MT SEM & RT Mean & RT SEM \\
\hline & B1 & 2.29 & 0.99 & 1091 & 41 & 316 & 14 \\
\hline & B2 & 1.88 & 1.11 & 911 & 41 & 272 & 9 \\
\hline & A1 & -36.55 & 1.12 & 1340 & 18 & 272 & 12 \\
\hline & $\mathrm{A} 2$ & -25.16 & 1.88 & 1228 & 25 & 262 & 11 \\
\hline & A3 & -15.49 & 1.55 & 1172 & 27 & 252 & 9 \\
\hline & A4 & -10.15 & 2.03 & 1091 & 31 & 256 & 11 \\
\hline & A5 & -12.51 & 1.82 & 1139 & 22 & 252 & 11 \\
\hline & A6 & -4.74 & 1.90 & 1054 & 30 & 245 & 8 \\
\hline & A7 & -5.64 & 1.54 & 1049 & 27 & 240 & 7 \\
\hline & A8 & -4.83 & 2.08 & 1028 & 26 & 241 & 7 \\
\hline & B3 & 15.14 & 1.98 & 1082 & 35 & 242 & 11 \\
\hline & B4 & 5.96 & 1.27 & 946 & 42 & 252 & 9 \\
\hline & B1 & 2.29 & 0.85 & 893 & 42 & 283 & 11 \\
\hline & B2 & 1.68 & 1.09 & 812 & 35 & 274 & 10 \\
\hline & A1 & -27.51 & 2.25 & 1259 & 23 & 252 & 12 \\
\hline & $\mathrm{A} 2$ & -16.84 & 2.00 & 1097 & 22 & 241 & 10 \\
\hline & A3 & -9.76 & 1.80 & 1078 & 26 & 245 & 10 \\
\hline & A4 & -8.13 & 1.75 & 1087 & 27 & 241 & 8 \\
\hline & A5 & -13.27 & 1.55 & 1083 & 28 & 249 & 6 \\
\hline & A6 & -7.75 & 1.13 & 1046 & 31 & 245 & 8 \\
\hline & A7 & -7.34 & 1.13 & 975 & 28 & 256 & 9 \\
\hline & A8 & -5.56 & 0.78 & 1006 & 36 & 247 & 10 \\
\hline & B3 & 17.05 & 2.01 & 1100 & 39 & 242 & 9 \\
\hline & B4 & 7.62 & 2.18 & 979 & 38 & 248 & 9 \\
\hline
\end{tabular}

John Wiley \& Sons, Inc.

This article is protected by copyright. All rights reserved. 
Table III. MNI coordinates of the suprathreshold clusters showing a significant correlation across participants between the intrinsic connectivity contrast and adaptation rate. In the direction column, - denotes that a more negative decay constant (i.e, faster adaptation) was associated with stronger connectivity between the suprathreshold cluster with the rest of the brain.

\begin{tabular}{llcccc}
\hline \multicolumn{1}{c}{ Phase } & Anatomic location & Direction & Coordinates of peak & T-value & Cluster size \\
\hline Early adaptation & L insular cortex & - & $-36,-12,10$ & 5.21 & 153 \\
Aftereffects & R central operculum & - & $48,-6,8$ & 4.47 & 96 \\
\hline \hline
\end{tabular}
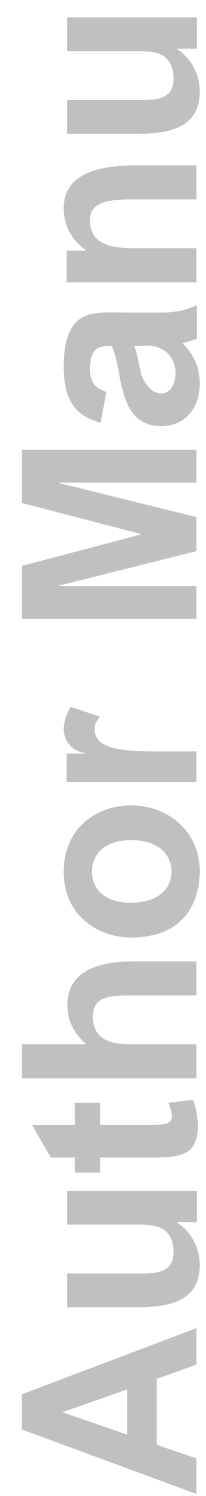

John Wiley \& Sons, Inc. 
Table IV. MNI coordinates of the suprathreshold clusters showing a significant correlation across participants between the gray matter volume and adaptation rate. In the direction column, denotes that a more negative decay constant (i.e, faster adaptation) was associated with larger gray matter volume in the suprathreshold clusters.

\begin{tabular}{llcccc}
\hline \hline Phase & Anatomic location & Direction & Coordinates of peak & T-value & Cluster size \\
\hline Early adaptation & L central operculum & - & $-45,-16,18$ & 6.43 & 2135 \\
& Precuneus cortex & - & $-4,-79,39$ & 5.06 & 1136 \\
& L postcentral gyrus & - & $-36,-37,48$ & 4.65 & 696 \\
Late adaptation & Cerebellum vermis VI & - & $-3,-79,-17$ & 5.30 & 863 \\
\hline \hline
\end{tabular}

John Wiley \& Sons, Inc.

This article is protected by copyright. All rights reserved. 